\title{
Schistosomiasis: Clinical Management of Liver Disease
}

\author{
Marta Guimarães Cavalcanti, M.D., Ph.D., ${ }^{*,}$ João Marcello de Araujo-Neto, M.D., ${ }^{\dagger}$ \\ José Mauro Peralta, M.D., Ph.D. ${ }^{\neq}$
}

Schistosoma infection is one of the most important causes of noncirrhotic portal hypertension in Latin America, Africa, and Asia. ${ }^{1}$ Schistosomiasis is a waterborne disease caused by a blood fluke of the genus Schistosoma. Schistosoma mansoni and S. japonicum are the species most commonly involved in liver disease. Schistosoma intercalatum, S. mekongi, and, occasionally, S. haematobium may also induce liver disease. ${ }^{2}$ Despite some species-specific variations in inflammatory/fibrotic responses, Schistosoma-induced liver injury results from a granulomatous inflammatory reaction around trapped Schistosoma eggs in the presinusoidal periportal spaces. In early phases of infection, a predominantly hypercellular nonfibrotic granuloma response produces liver dysfunction that is not clinically detectable. Imaging studies may reveal enlargement of the left liver lobe without changes in the liver parenchyma or splenomegaly. Reversibility of these findings is expected in 12 months following chemotherapy. ${ }^{3}$ Development of chronicity results in collagen deposition in the periportal spaces, which is the basis of the pathognomonic pathological feature of schistosomalassociated liver fibrosis known as "Symmers' pipestem fibrosis". In addition, fibrosis is accompanied by angiogenesis. Vascular disturbances include severe reduction and distortion of the portal venous system and hyperplasia and hypertrophy of the arterial system. ${ }^{4}$ The occlusion of the portal veins is associated with portal hypertension marked by splenomegaly, portocaval shunting, and gastrointestinal varices. Ultrasonography (US) may reveal periportal fibrosis around periportal spaces, liver parenchymal heterogeneity, splenomegaly, enhanced portal vein dimensions, and the presence of collateral vessels. Despite successful parasite elimination, the effect of chemotherapy on the inflammatory response within the liver is negligible. As the fibrosis progresses, with or without active infection, repeated episodes of variceal bleeding may be accompanied by hepatic deterioration that can lead to a fatal outcome. Worsening of liver function might also be provoked by Schistosoma-associated coinfections or other comorbidities. Viral hepatitis, malaria, HIV infection, mixed Schistosoma species, alcoholism, or nonalcoholic steatohepatitis each may enhance tissue damage and/or inflammatory/fibrotic responses, thus promoting disease progression. ${ }^{2,3}$

\section{Diagnosis of Schistosoma Infection and Liver Staging}

A stepwise approach to the clinical management of Schistosoma liver disease includes detection of active Schistosoma infection by using accurate diagnostic tools, assessment of hepatic morbidity and disease severity, followed by antihelminthic therapy with praziquantel (PZQ). The diagnosis of schistosomiasis at any phase of infection (acute or chronic) is based on demonstration using laboratory investigations of Schistosoma infection. The assessment of the morbidity associated with schistosomiasis is usually accomplished in both community and institutional settings by clinical assessment, laboratory tests, and imaging tests (Table 1). PZQ is the drug of choice to treat laboratory-proven schistosoma infection.

Traditionally, demonstration of parasite eggs in feces, urine, or tissue confirms Schistosoma infection. However, in the case of Schistosoma liver disease, detection of ova by parasitological tests and biopsy often fail to demonstrate active

Abbreviations: PZQ, praziquantel; US, ultrasonography.

From the *Infectious and Parasitic Diseases Service, Hospital Universitário Clementino Fraga Filho, Universidade Federal do Rio de Janeiro, Rio de Janeiro, Brazil, ${ }^{\dagger}$ Department of Clinical Medicine, Faculdade de Medicina, Universidade Federal do Rio de Janeiro, Rio de Janeiro, Brazil, ${ }^{\ddagger}$ Department of Immunology, Instituto de Microbiologia Paulo de Góes, Universidade Federal do Rio de Janeiro, Rio de Janeiro, Brazil

Potential conflict of interest: Nothing to report.

View this article online at wileyonlinelibrary.com

(C) 2015 by the American Association for the Study of Liver Diseases

doi: 10.1002/cld.495 
TABLE 1 Assessment of Schistosomiasis: Diagnosis, Disease Staging with Morbidity Markers, and Follow-Up Post-Treatment

\begin{tabular}{|c|c|c|c|}
\hline Assessment & & $\begin{array}{l}\text { Community } \\
\text { Settings }\end{array}$ & $\begin{array}{l}\text { Institutional } \\
\text { Settings }\end{array}$ \\
\hline \multirow[t]{2}{*}{ Diagnosis } & Traditional & $\begin{array}{l}\text { Parasitological } \\
\text { methods }\end{array}$ & $\begin{array}{l}\text { Parasitological } \\
\text { methods } \\
\text { Biopsy*/tissue }^{\dagger} \\
\text { Serology }\end{array}$ \\
\hline & New Tools & $\begin{array}{l}\text { Immunodiagnosis } \\
\text { DNA detection } \\
\mathrm{RT}^{\ddagger}\end{array}$ & $\begin{array}{l}\text { Immunodiagnosis } \\
\text { DNA detection } \\
\mathrm{RT}^{\ddagger}\end{array}$ \\
\hline \multirow[t]{2}{*}{$\begin{array}{l}\text { Morbidity } \\
\text { Markers }\end{array}$} & Traditional & $\begin{array}{l}\text { US } \\
\text { FOB }\end{array}$ & $\begin{array}{l}\text { Doppler imaging } \\
\text { Endoscopy } \\
\text { Colonoscopy } \\
\text { FOB }\end{array}$ \\
\hline & New Tools & - & $\begin{array}{l}\text { Computed tomography } \\
\text { Magnetic resonance } \\
\text { Liver elastography }\end{array}$ \\
\hline \multirow[t]{2}{*}{$\begin{array}{l}\text { Follow-Up } \\
\text { Post- } \\
\text { Treatment }\end{array}$} & Traditional & $\begin{array}{l}\text { Parasitological } \\
\text { methods } \\
U^{\dagger \dagger}\end{array}$ & $\begin{array}{l}\text { Parasitological } \\
\text { methods } £ \\
\text { US }^{\dagger \dagger} \\
\text { Doppler imaging } \\
\text { Endoscopy }^{\dagger \dagger} \\
\text { Colonoscopy }\end{array}$ \\
\hline & New Tools & $\begin{array}{l}\text { DNA detection" } \\
\text { RT }^{\#}\end{array}$ & $\begin{array}{l}\text { DNA detection } \\
\text { RT }^{\#} \\
\text { Liver elastography }\end{array}$ \\
\hline
\end{tabular}

Community settings is applicable to endemic areas.

Abbreviations: FOB, fecal occult blood; RT, rapid test; US, ultrasonography.

*Biopsy: Includes rectal snips and liver biopsy

${ }^{\dagger}$ Tissue: surgical specimens

${ }^{\ddagger}$ Rapid Tests: point-of-care CCA and CAA (S. mansoni)

$\S$ In individuals who are not egg excretors before treatment, parasitological tests are unsuitable for follow-up and assessment of cure

${ }^{\dagger \dagger}$ Indicated in individuals with hepatic schistosomiasis

"In individuals with concomitant intestinal presentation.

\#Few numbers of studies and/or small study populations recruited are the limitations to validate the procedures. Further studies are still necessary.

infection. Diagnosis of schistosoma liver disease in patients who are non-egg excretors requires alternative diagnostic tools such as immunodiagnosis (serology) and DNA-based assays. ${ }^{5}$ Furthermore, assessment of drug response by egg detection may underestimate rates of drug failure due to persistence of infection (without egg elimination) or reinfection. Although serology may remain positive for long periods after chemotherapy has been completed, $\cong 30 \%$ of reactive individuals with active infection become antibodynegative in response to therapy (ie, seroconvert). Therefore, seroconversion suggests an efficacious response to therapy. When DNA-based assays are used, the persistence of DNA suggests failure to eliminate Schistosoma infection and no cure. $^{6}$ Point-of-care (POC) platforms, such as rapid tests to detect cathodic circulating antigen (CCA) and anodic circulating antigen (CAA), have been used in community settings for surveillance purposes. Availability of commercial kits and easy-to-do tests are quite appealing for use in both community and clinical settings. Accuracy of POC-CCA (or "rapid tests") is comparable or slightly superior to Kato-Katz test in high and moderate prevalence areas where infections with high parasite loads are very common. ${ }^{7,8}$ However, these assays carry the risk of underdetection of active infection in individuals with no or low egg excretion, such as in immigrants and travelers who have been recently infected. Use of rapid tests as a marker of drug response is still debatable.

In addition to improved strategies for diagnosis of Schistosoma infection, assessment of morbidity is essential. Imaging tests allow liver disease staging, while also serving as decision-making examinations (Fig. 1). US, for instance, is central to liver morbidity determination. ${ }^{9,10}$ US is one of the most accessible tools for determining disease severity and is affordable even in remote areas.

\section{Clinical Management}

Schistosomiasis has a broad spectrum of clinical presentations, and up to $10 \%$ of patients may have severe hepatosplenic presentation. Although severe forms of disease are expected to correlate with high intensity of infection, which are commonly seen in areas of high and moderate endemicity, individuals living in low endemic and nonendemic areas may also present with advanced liver disease, even without egg excretion. Therefore, the overriding concern in care of the patient with Schistosoma liver disease is the proper management of the different stages of hepatic injury arising from the chronic inflammatory response to current or previous Schistosoma ova antigens (Fig. 1). Improvement of staging and follow-up of liver disease depends on determination of morbidity levels by biological markers and imaging diagnostic tests.

PZQ is indicated to treat laboratory-proven schistosoma infection. A single dose of PZQ $40 \mathrm{mg} / \mathrm{kg}$ is effective in $S$. mansoni infection. However, regimens may vary depending on the species involved, individual age, and even the site of infection. In addition, some individuals with residual infection or after re-exposure may require a second course of treatment. Furthermore, after eradication of Schistosoma infection with chemotherapy, some individuals demonstrate persistence of the inflammatory response and progression to advanced stages of liver disease, such as portal hypertension. Traditionally, the post-therapy response is determined by parasitological methods 4 to 6 weeks after PZQ administration (Table 1). Monitoring the post-therapy response may require repeated testing after 6 and 12 months if residual schistosomiasis or reinfection is an issue. Tissue biopsy or surgical samples obtained by invasive procedures are usually unsuitable for long-term follow-up. Assessment of the drug response in non-egg excretors will require alternative methods such as DNA 


\section{Schistosomiasis Clinical Management}

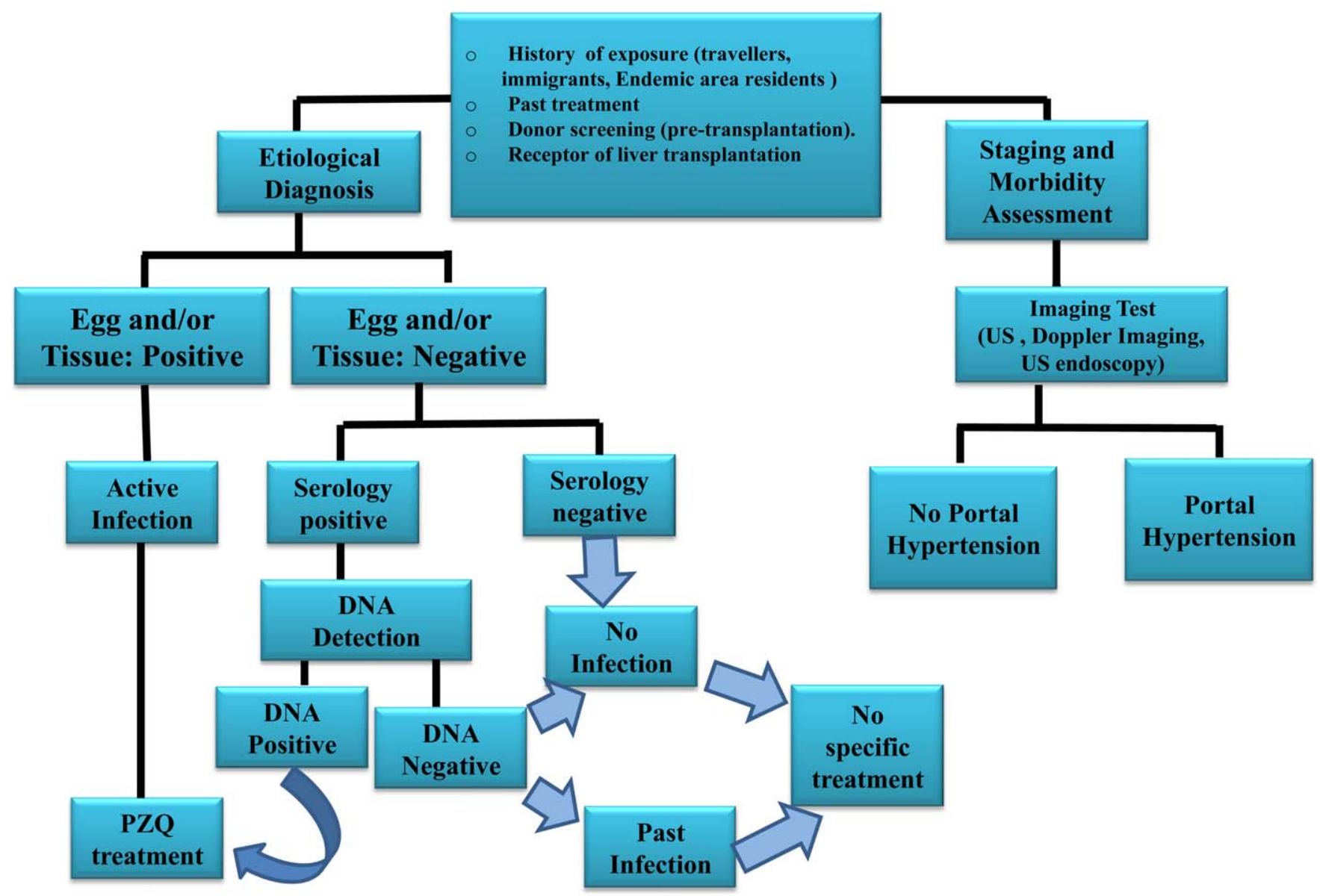

Figure 1 Algorithm showing the clinical management of schistosomiasis. "Egg and/or tissue" indicates detection of eggs by parasitological methods and/or tissue biopsy (or surgical specimen). "Past infection" is defined as persistent immunoreactivity in the absence of egg and/or DNA detection with or without previous confirmed treatment. Abbreviations: PZQ, praziquantel; US, ultrasonography.

detection. Although DNA detection (or rapid test) more accurately identifies active infection after PZQ use, no standard protocols currently include those methods. In chronic hepatosplenic forms of S. mansoni infection, liver and spleen size may achieve around 80\% reduction after PZQ use as measured by conventional US in a period of 1 to 5 years. ${ }^{11}$ However, persistence of inflammatory process also occurs for prolonged periods (several years) in some cases. New anti-Schistosoma drugs and inhibitors of fibrosis have been investigated, but their routine use is still far from daily practice. However, this prediction might change rapidly with the elucidation of gene regulation involved in disease progression, thereby opening new horizons on disease staging, therapy, and clinical management of Schistosoma liver disease. ${ }^{12}$ Moreover, the presence of comorbidities such as coinfections with viral hepatitis, the use of immunosuppressive drugs, and organ transplantation pose new challenges.

\section{Conclusion}

Schistosomiasis remains an important cause of liver disease in areas of frequent transmission and presents as a challenge to diagnosis in nonendemic areas. Detection of active infection and staging of liver disease are the main goals in schistosomiasis management. Unfortunately, traditional approaches are often successful in both diagnosis and morbidity assessment. New diagnostic approaches, standardization of imaging tests, and development of new investigative technologies are changing this scenario.

\section{CORRESPONDENCE}

Peralta, JM, Departmento de Imunologia, Instituto de Microbiologia Paulo de Góes, Universidade Federal do Rio de Janeiro, Rio de Janeiro, Brazil.

E-mail: peralta@micro.ufrj.br 


\section{References}

1. Bodh V, Chawla YK. Noncirrhotic intrahepatic portal hypertension. Clin Liver Dis 2014;3:129-132.

2. Koukounari A, Donnelly CA, Sacko M, Keita AD, Landouré A, Dembelé R, et al. The impact of single versus mixed schistosome species infections on liver, spleen and bladder morbidity within Malian children pre- and postpraziquantel treatment. BMC Infect Dis 2010;10:227.

3. Mazigo HD, Nuwaha F, Wilson S, Kinung'hi SM, Morona D, Waihenya R, et al. Epidemiology and interactions of Human Immunodeficiency Virus - 1 and Schistosoma mansoni in sub-Saharan Africa. Infect Dis Poverty 2013;2(1):2.

4. Pereira TA, Xie G, Choi SS, Syn WK, Voieta I, Lu J, et al. Macrophage-derived Hedgehog ligands promotes fibrogenic and angiogenic responses in human Schistosomiasis mansoni. Liver Int 2013;33:149-161.

5. Cavalcanti MG, Silva LF, Peralta RH, Barreto MG, Peralta JM. Schistosomiasis in areas of low endemicity: a new era in diagnosis. Trends Parasitol 2013;29: $75-82$.

6. Downs JA, Kabangila R, Verweij JJ, Jaka H, Peck RN, Kalluvya SE. Detectable urogenital schistosome DNA and cervical abnormalities 6 months after single-dose praziquantel in women with Schistosoma haematobium infection. Trop Med Int Health 2013;18:1090-1096.
7. Tchuem Tchuenté LA, Kueté Fouodo CJ, Kamwa Ngassam RI, Sumo L, Dongmo Noumedem C, Kenfack CM, et al. Evaluation of circulating cathodic antigen (CCA) urine-tests for diagnosis of Schistosoma mansoni infection in Cameroon. PLoS Negl Trop Dis 2012;6:e1758.

8. Coulibaly JT, N'Gbesso YK, Knopp S, N'Guessan NA, Silué KD, van Dam GJ, et al. Accuracy of urine circulating cathodic antigen test for the diagnosis of Schistosoma mansoni in preschool-aged children before and after treatment. PLoS Negl Trop Dis 2013; 7:e2109.

9. Lambertucci JR. Revisiting the concept of hepatosplenic schistosomiasis and its challenges using traditional and new tools. Rev Soc Bras Med Trop 2014; $47: 130-136$

10. el Scheich T, Holtfreter MC, Ekamp H, Singh DD, Mota R, Hatz C, et al. The WHO ultrasonography protocol for assessing hepatic morbidity due to Schistosoma mansoni. Acceptance and evolution over 12 years. Parasitol Res 2014;113:3915-3925.

11. Domingues AL, Coutinho AD. Reduction of morbidity in hepatosplenic schistosomiasis mansoni after treatment with praziquantel: a long term study. Rev Soc Bras Med Trop 1990;23:101-107.

12. Dessein A, Arnaud V, He H, Li J, Dessein H, Hou X, et al. Genetic analysis of human predisposition to hepatosplenic disease caused by schistosomes reveals the crucial role of connective tissue growth factor in rapid progression to severe hepatic fibrosis. Pathol Biol (Paris) 2013;61:3-10. 